\title{
Systematic Approach for Development of Knowledge Base in Higher Education
}

\author{
Teba Fadhil Muhsin \\ Anjum Zameer Bhat \\ Imran Ahmed \\ Mohamed Samiulla Khan
}

Internet of Things and Big Data are revolutionary technologies bringing innovation in almost every sector. These innovative technologies have of late been implemented in the education sector with significant success in achieving the goal of "education par excellence". A lot of research has been conducted to yield the benefits of IoT in the education sector and many models have been proposed in the past by various researchers all around the world. This research introduces a systematic manner of collecting the data using sensing devices from various cohorts of students studying at "Middle East College" and how this data can be utilized to infer various postulations related to the enhancement of teaching and learning. Moreover, visualization of the facts that may provide preventive and preemptive capabilities to manage and support them in decision making is also explored in great detail. This research work is an effort to contribute to the development of a strong educational system through innovation and inquiry. This research work is also intended to contribute to developing the foundation of "knowledgebase" for Middle East College.

\section{Introduction}

IT has revolutionized the modern world by providing unprecedented automation, prompt services, improved communication and access to the sea of information. We are living in a century which has witnessed big leaps in technology, innovation, communication and automation. Although we have cutting edge technologies at disposal however a lot needs to be done in order to apply these innovative technologies to variety of fields. Innovation in many areas is the need of the time and any enterprise irrespective of a particular field of expertise is going to lose its significance if it is not able to embed innovation and innovative technologies of 21st century. Internet of Things and Big Data have proven to be one amongst few impactful, significant and best innovations of the 21st century. Internet of Things has entirely changed the perception of the people about the limitations of the technology. Big Data analytics has given ability to see the invisible which brings an unparalleled capability for organizations to achieve impossible. These innovative technologies need to be explored and exploited to the maximum extend to achieve technology revolution which is beyond common human perception. The Internet of Things (IoT) and Big Data has witnessed an exponentially growing demand for implementation in various critical and significant fields like healthcare, Transportation, Agriculture, aviation, marketing, retail business, ecommerce and education. In education the implementation of Big Data and IoT is relatively less and gradual however surely growing.

A developed country has a strong education system, education is one the most essential investments a country can make in its people and its future. The field of education should be the one which accumulates most of the innovations and innovative technologies for the enrichment of teaching and learning. There is a clear evidence of implementation of IoT and Big Data in education sector or at-least research studies which showcase the framework for such an implementation [1] [2]. This research work showcases the implementation of IoT and Big Data in education sector for enhancement of teaching and learning and for providing vital insights to the administrators and policy makers to appropriately plan the future course of action. Moreover, this innovative step 


\section{Journal of Student Research}

would lead to creation of knowledgebase for higher education institutions.

This research exhibits the stepwise and procedural collection of data from students of higher education institution. The data collection testing is being conducted in Middle East College.This research work shows how useful data can be collected from various cohorts of students in a higher education institution using advanced sensors and how this data can be translated into useful information for the benefits of an higher education institution. The data is collected with a wearable device called electroencephalograph (EEG). Ten cohorts of bachelors and diploma students are shortlisted for collection of data. The cohorts are belonging to various levels in different years of study so that numerous inferences can be generated from data collected. The wearable device is made available to a particular cohort and a specific method or pedagogy of teaching is practiced. Electroencephalograph (EEG) is capable of monitoring brain activity by measuring voltage fluctuations resulting from ionic current within the neurons of the brain [3]. There are various wearable devices that can be used to monitor the activity of the bran. In a particular cohort, the students are monitored with a particular teaching method or pedagogy practiced. In same session various types teaching methods are practiced during various sessions and the brain activity of the students is monitored through the wearable EEG device. The same method of teaching are repeated in many of the sessions and an average brain activity value is taken for each of the teaching and learning methods. Similar kind of activity is conducted on 10 different cohorts at various levels of undergraduate and diploma programs. The sensing devices that are worn by the students are connected to the aggregator that aggregates the signals that are received from various sensors which are used by the students. The functionality of the aggregator is to collect the data in an efficient manner and forward it to the storage device for storing and later processing and analyzing. Electroencephalography is a device used to sense the brain's electrical activity. The EEG headset device has built-in electrodes sensors, these electrodes will sense and collect the electrical signals of the brain neurons when placing the device on the student's surface, it will basically sense the changes occurrence in the brain's voltage due to the movement of the ionic inside the brain [4]. The activity of the brain during various sessions of teaching with different methods can provide us with a pattern which can lead to various inferences as follows.

- A teaching method most suitable for a particular subject/topic and level of students.

- Comparison of various teaching methods and their impact on student learning.

- Most suitable levels, topics and subject for a particular teaching method.

- Effectiveness of various teaching practices.

- Benefits of bringing minor modifications to a particular teaching method.

- Benefits of innovative teaching practices.

The teaching methods that were practiced in various sessions will be provided with a value associated to the average attention levels students had during the session of the class. The average of this value for various session for each teaching practice is taken as its final value to determine the effectiveness of a particular teaching method. Moreover, effectiveness of same teaching method in different environments is also calculated. This operation provides us with various inferences and conclusions e.g. most effective teaching method for comprehending OSI reference model or most effective method to understand the differential equation. The brain activity is taken as the indicator for level understanding of a particular concept. The activity of brain is converted in to a pattern that is measured on a scale of 5 which indicates the attention level or concentration level of students during a particular session. It also gives an indication that for how long with a particular teaching method the concentration levels of students were high and when it started decreasing considerably moreover it can provide an average time period after which the concentration levels abysmally diminished. This information is very critical for the enhancement of teaching and learning as when this information is visualized it can provide an academician with very precise and important inputs which would be beneficial for management of his class and better teaching and learning experience. As an example, a teacher can get tentative idea of the time slot he/she can maximum deliver a session with a specific method of teaching beyond which the concentration levels of the students diminish. Teacher can also can an idea about which teaching methods are 


\section{Journal of Student Research}

Fourth Middle East College Student Research Conference, Muscat, Sultanate of Oman

more suitable for a specific subject area and topics and by following those teaching methods teacher may get maximum response from students as far as their understanding of the concepts is concerned. The changes that are brought to one's delivery methods like innovative ways of teaching, flipped teaching, student centered teaching and learning etc. can be measured and inferences can be drawn about which particular way or method in a given circumstances are more suitable and which ones may not have much of the impact. By implementing this concept of continuously monitoring the concentration levels of students and various other parameters can be later on added to draw more inferences and hidden facts. As an example, the grades of students at the end of the semester can be compared to their attend levels in the classrooms and predictive analysis can be done to establish the likely scores of students who have a particular level of concentration in the classroom. Moreover, the different ways can be adapted to ensure that concentration of students is increased during a teaching session. This way of monitoring may also help student success center at the college level to understand individual problem of students in a much better manner. This can also help them to address the problems and see the change or development in the students over a period of time.

\section{Methodology}

\section{Data Collection with EEG}

EEG headset collects the electrical brain activity of a student/human once the headset is placed on their head surface, the electrical brain activity gets generated when ions are being interchanged regularly between the huge number of neurons and the extracellular milieu inside the human brain. All of the ions that have the same charge they tend to repulse one another, which will result in a

huge number of ions repelling each other at the same period of time, and thus they tend to push away their neighbor ions, who would do the same action to their neighbors and so and so until this process will end up creating a wave.

Having an individual neuron that generates a single electrical potential cannot be captured by an EEG headset because it has a very small size and thus the collected EEG waves are the addition of hundreds, and millions of the same spatial orientation neurons.

EEG will show oscillations that have different ranges of frequencies each of them describes a specific function of the brain, these in return will create networks of neurons that represent various of synchronized activities.

In terms of collecting data related to the student's brain activity during the time of taking a class the EEG will collect the data related to the "Cerebral Cortex" of the brain, this cortex has many functions such as "Intelligence Determination, Understanding, Motor Function, and Processing of Language", this cortex has sensory and motor areas, the sensory areas are in charge of receiving information and processing these information into a sensed data [10] . 


\section{Journal of Student Research}

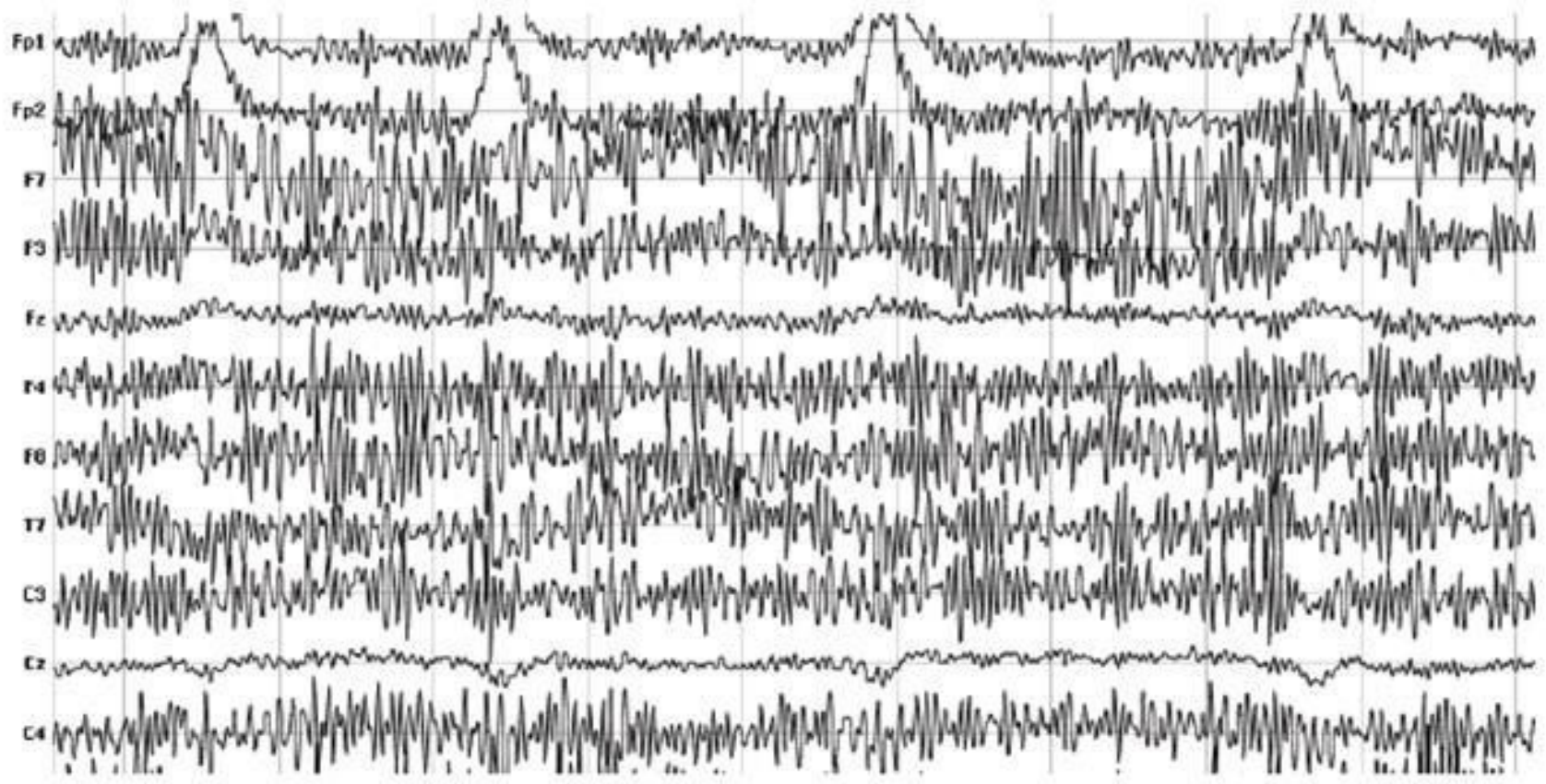

Figure 1. Example of EEG Data.

\section{Knowledge Base for Higher Education establishments: an architecture}

The systematic development and implementation of IoT using wearable devices in the educational establishments would result in the creation of a knowledge base that can be utilized for variety of purposes by the educational institution ranging from improvement in teaching and learning, student assistance and support, administration and management, future planning, forecasting and predictive analysis and enhancement of student performance. The knowledge base has five significant modules. Each module has a specific function to perform and contributes to the overall development of the knowledge base for the higher education institution. The following figure shows various modules of the knowledge base and reflects the main functionality of each module.

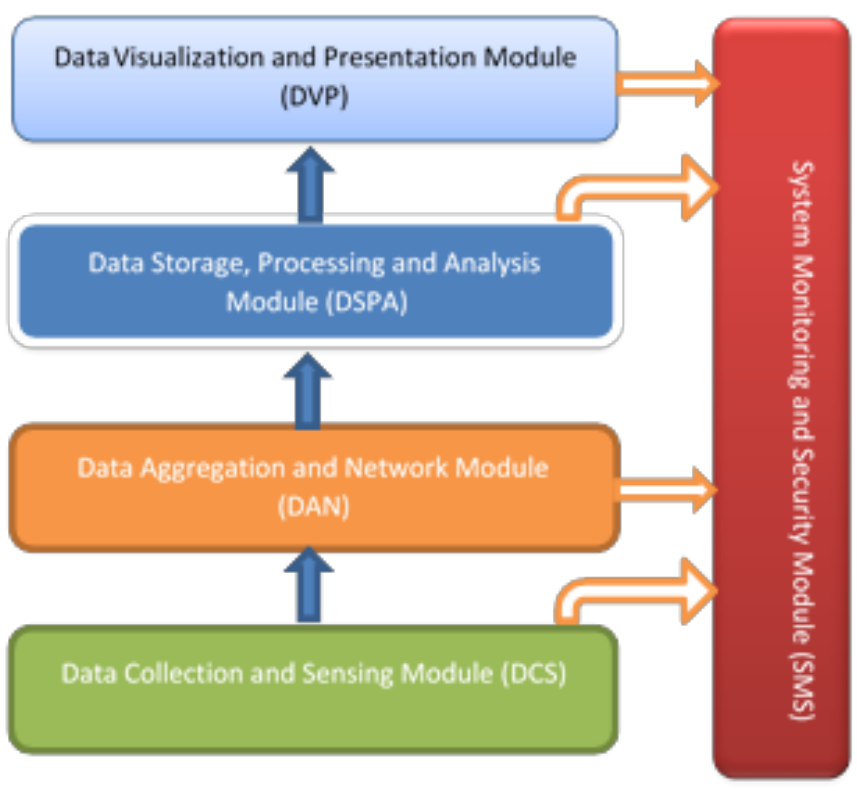


Figure 2. Modular architecture for Knowledge base for Higher Education Institutions

Fig. 2. depicts the modular architecture of the knowledge base that can be developed for education establishments. The architecture is having five modules. Starting from the bottom, Data Collection and Sensing Module (DCS) provides the knowledge base with capabilities of generating huge amount of data that is collected from various sensing devices, EEG innovative wearables, smart tables, information systems, smart document cameras and other new and innovative devices. The functionality of this module is to collect the appropriate data in huge quantity from various connected devices and send it in to the Data Aggregation and Network Module (DAN). The following figure Fig. 3 depicts the main components of the Data Collection and Sensing Module (DCS) and some of the significant devices that can be a part of this Module. The depicted figure shows few of the main devices that can be used however all the sensing and data collection devices are not shown in the figure.

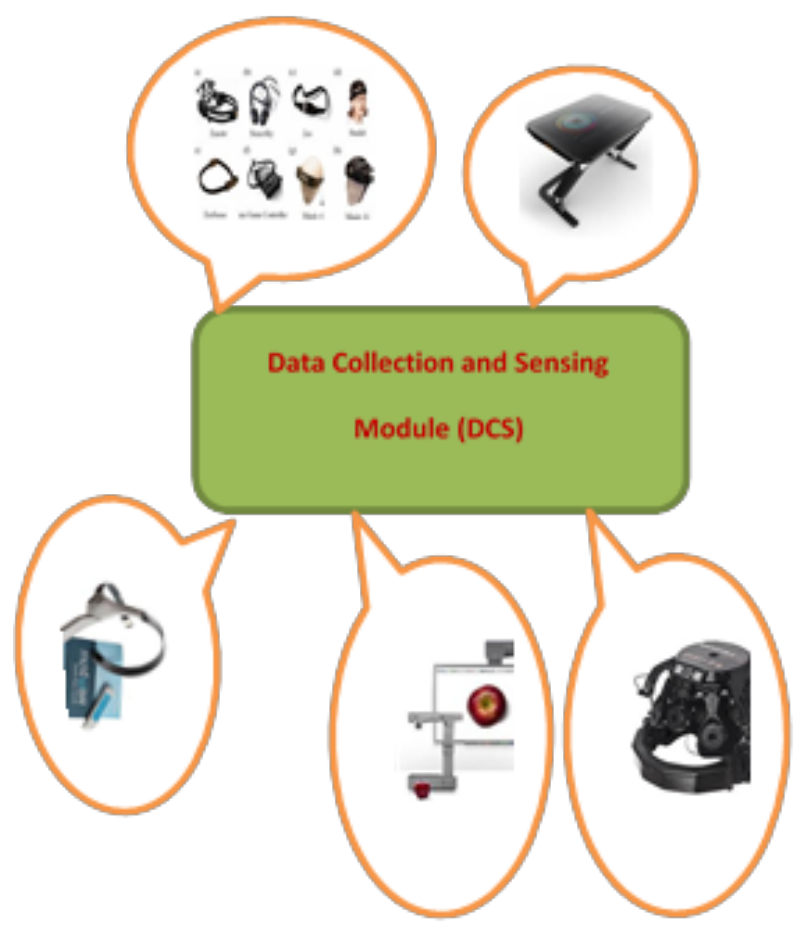

Figure 3. Data Collection and Sensing Module (DCS)

The above figure Fig. 3 shows various devices that are a part of Data Collection and Sensing Module (DCS). The figure depicts various wearable devices that can be used as sensors and fetch huge amount of data. Most of these devices are easy to wear and can be provided to students in the class rooms without any difficulty. Most the devices which are wearable are well under the budget and can be purchased for a specific cohort of students. The figure also shows a smart table and an HD document camera which can also be used for fetching the useful data. Various headset which are actually the Electroencephalography (EEG) readers are used to monitor the brain activity. The second module of the architecture is data aggregation and network module. This module consists of aggregators that are having capability to receive and aggregate the signals from various sensors for e.g. raspberry pi. This module also consists of high-end network devices and protocols which in combination can provide seamless data transfer between Data Aggregator and Network Module (DAN) and Data Storage, Processing and Analysis Module (DSPA). This layer is depicted in the following figure Fig. 4. 


\section{Journal of Student Research}

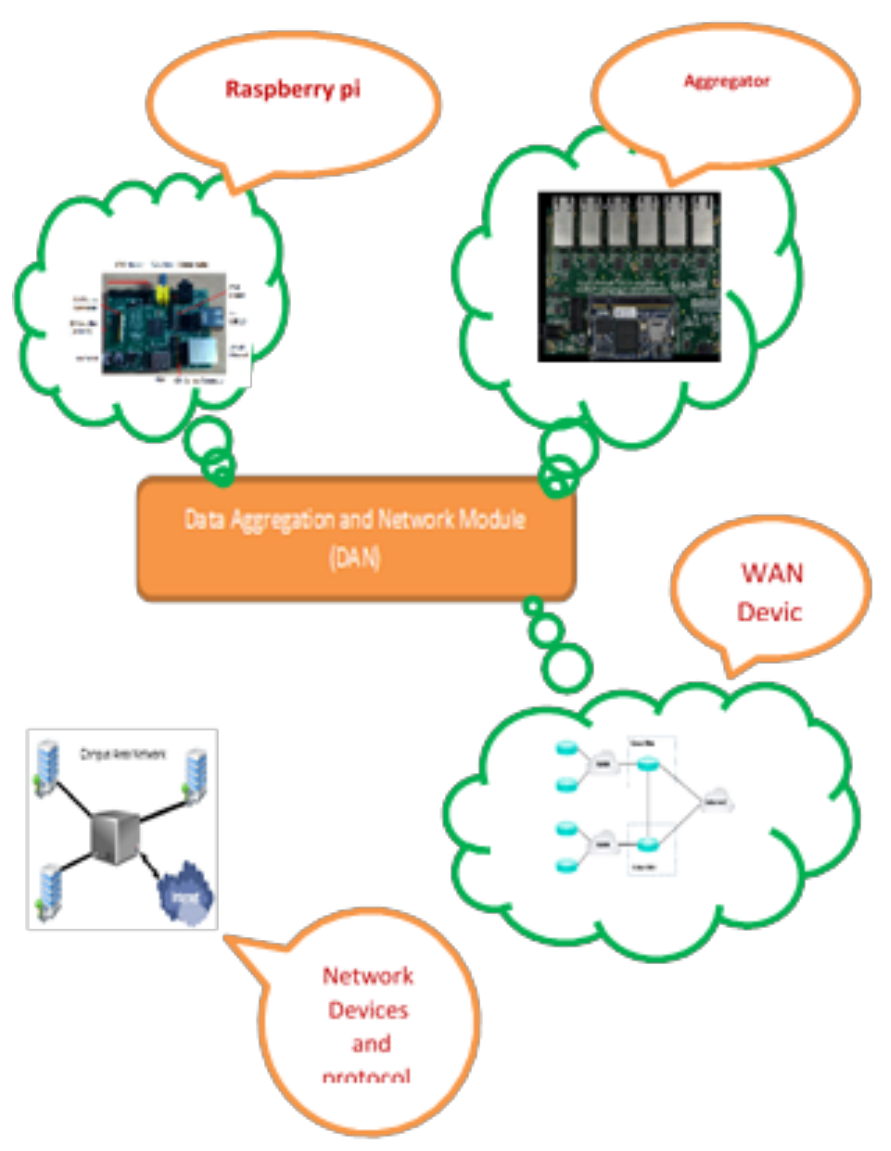

Figure 4. Data Aggregation and Network Module (DAN)

Fig. 4 Data Aggregation and Network Module (DCS) Data aggregation and network module (DAN) comprises of various WAN devices and aggregators that are used to aggregate the signals that are collected from various sensors and other data collection devices. The Fig. 4 shows Raspberry pi as one of the aggregators used in the system. The third Module of the knowledge base consists of the Data Storage, Processing and Analysis Module (DSPA), this module is responsible for storage of the huge amount of data that is collected from the network layer. This layer typically is a cloud which provides facilities for storage, retrieval, processing and analysis of the big data. This is one of the most important Modules of the knowledge base which provides the capabilities for predictive analysis and consists of the devices, servers, applications, databases having capability of big data storage and analytics. This Module can be hired from a public cloud service provider or can be established by implementing a private cloud by the educational establishment. Fig. 5 shows the typical Data Storage, Processing and Analysis Module (DSPA) and its components [11]. 


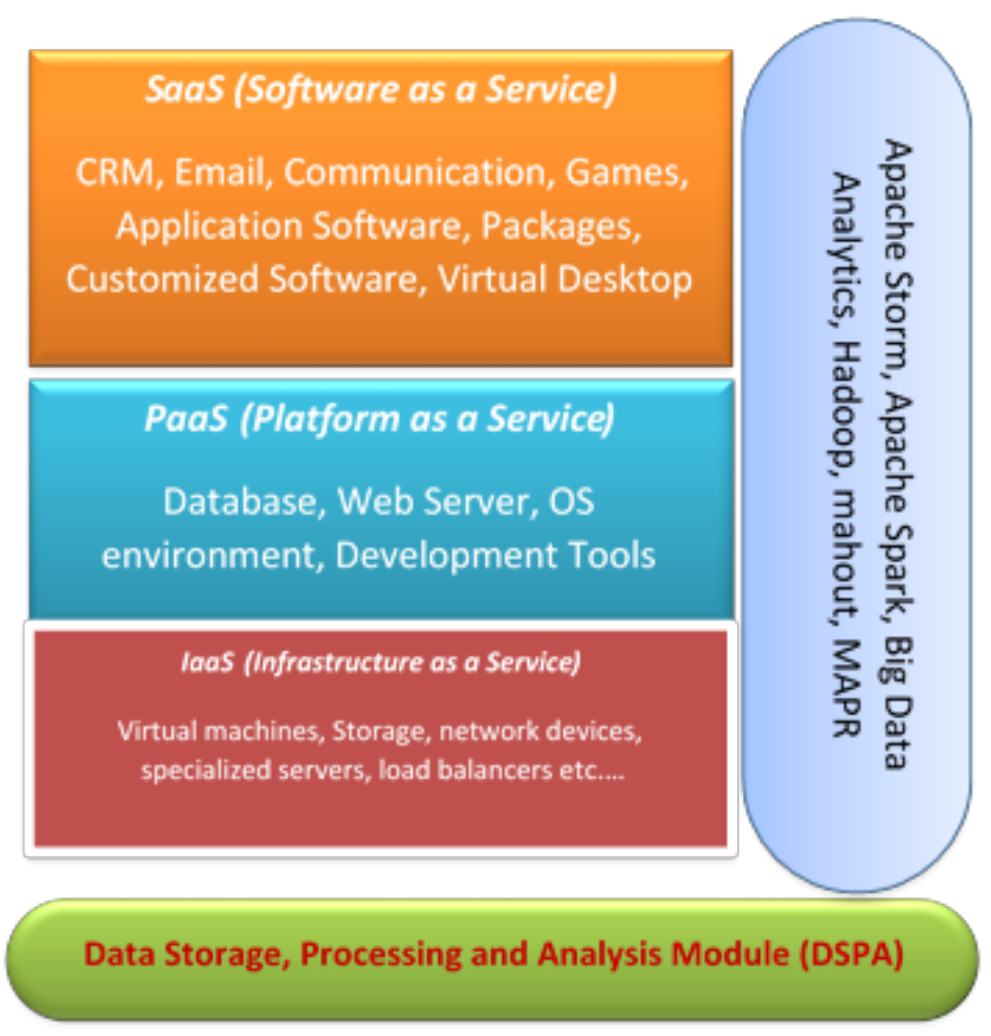

Figure 5. Data Storage, Processing and Analysis Module (DSPA)

The above figure Fig. 5 depicts the typical architecture of the Data Storage, Processing and Analysis Module (DSPA). The fourth Module of the architecture Data Visualization and Presentation Module (DVP) provides with application that are capable of presenting the processed data in the desired manner. This module is equipped with the applications that are capable of visualization, presentation, drilling down capabilities etc. This Module fetches the processed information from the Data Storage, Processing and Analysis Module (DSPA) and makes it presentable for the users to see the results. The Module 5 of the architecture for knowledge base is the System Monitoring and Security Module (SMC) which applies various monitoring and security features to different modules e.g. it applies physical security to Data Collection and Sensing Module (DCS) by providing physical security and monitoring to sensing devices that are used for fetching the data. The DCS provides encryption to the Data Aggregation and Network Module (DCS) so that the data which is transferred through the network is not compromised and integrity and validity of data is not breached. System Monitoring and Security Module (SMC) provides the features of Data Privacy to Data Storage, Processing and Analysis Module (DSPA) so that data that is stored, processed and Analyzed on this module is not being leaked so that its privacy gets compromised. The System Monitoring and Security Module (SMC) provides the security to Visualization and Presentation Module (DVP) by enabling authentication and authorization features so that application access is provided to legitimate users with specific privileges. The System Monitoring and Security Module (SMC) is overall responsible for the security of the entire system from the onset of data acquisition (Module 1) to data presentation (Module 4).

\section{Conclusion}

Higher education institutions are the backbone of a nation which impact every realm and sphere having direct impact on economy, development and Quality of life. Higher education institutions in Sultanate of Oman and elsewhere in the world need transformation to embed new and innovative technologies to foster educational excellence. This research work is based on the same premise so as to provide a systematic approach for higher education establishments to develop a knowledge 


\section{Journal of Student Research}

Fourth Middle East College Student Research Conference, Muscat, Sultanate of Oman

base which can have a significant impact on the higher education establishment in terms of quality and governance. This research work is expected to contribute towards the development of better educational strategy in higher education institution as well as it is expected to impact the quality of teaching \& learning. This research work provides a guideline which would result in the development of a decision support system that can be used by the management of the higher education establishment to take better decisions. Moreover, the development of knowledge base provides preemptive and preventive capabilities to higher education establishments which can facilitates academic excellence in the future.

\section{Acknowledgements}

All praise and glory to Almighty for bestowing the ability and knowledge to conduct this research work. I thank Middle East College in Sultanate of Oman for their continued support and cooperation, I am thankful to the management of Middle East College for providing a conducive environment for research and for supporting and sponsoring research activities. I am thankful to Vivekananda Global University for their support and cooperation. I am also indebted to my supervisor for his painstaking efforts to support this work without whom it would not have been possible. I am thankful to all my friends, colleagues and family members who supported and encouraged me throughout this research work.

\section{References}

1. Z. Bhat and I. Ahmed, "Big Data for institutional planning, decision support and academic excellence," in MEC IEEE international Conference on Big Data and Smart City, Muscat, 2017.

2. J. Marquez, J. Villanueva, Z. Solarte and A.Garcia, "IoT in Education: IntegrationofObjectswithVirtual Academic Communities," New Advances in Information Systems and Technologies, vol. 444, pp. 201212, 2016.

3. N. E. and d. S. F.L, "Electroencephalography: Basic Principles, Clinical Applications, and Related Fields.," Lippincott Williams \& Wilkins. ISBN 978-07817-5126-1, 2004.

4. J. Desai, "'Electroencephalography (EEG) Data Collection and Processing through Machine Learning" (2014). Theses and Dissertations. 2160.," 2014.

5. M. BAGHERI and S. HAGHIGHI MOVAHED, "The Effect of the Internet of Things (IoT) on Education Business Model," Sheffield Hallam University, 2016.

6. M. Maksimović, "Green Internet of Things (G-IoT) at engineering education institution: the classroom of tomorrow," Infotec Johorina, vol. 16, 2017.

7. X. Nie, "Constructing Smart Campus Based on the Cloud Computing Platform and the," in Proceedings of the 2nd International Conference on Computer Science and Electronics Engineering (ICCSEE 2013), China, 2013.

8. Educause, "7 things you should know about wearable technology," ProQuest, vol. 35, no. 5, pp. 346-347, 2013.

9. G. Jayavardhana, B. Rajkumar, M. Slaven and P. Marimuthu, "Internet of Things (IoT): A vision, architectural elements, and future directions," Elsevier, vol. 29, no. 7, pp. 1645-1660, 2013.

10. R. Bailey, "Anatomy of the Brain: Cerebral Cortex Function.," thoughtco, 2018.

11. A. Z. Bhat, B. Singh and A. V. Singh, "Learning resources as a service (LraaS) for Higher Education Institutions in Sultanate of Oman," IEEE, Noida, 2017. 